\title{
Implementación del presupuesto de ventas para la optimización de los ingresos de la empresa maderera "Don Junior" en Santo Domingo, Ecuador
}

\section{Implementation of a sales budget for the optimization of the income of the wood company "Don Junior" in Santo Domingo, Ecuador}

DOI: $10.46932 / \mathrm{sfjdv2n3-071}$

Received in: May 1st, 2021

Accepted in: Jun 30th, 2021

\section{Sofía Elizabeth Quiguirí Udeo}

Ingeniera en Contabilidad y Auditoría, CPA, por la Pontificia Universidad Católica del Ecuador, Sede Santo Domingo, Ecuador

Institución: Pontificia Universidad Católica del Ecuador, Sede Santo Domingo, Ecuador

Dirección: Vía Chone Km 2, Santo Domingo, Ecuador, CP: 230203

E-mail: sequiguiriu@pucesd.edu.ec

\section{Maylee Lizbeth Salinas Ordoñez}

Ingeniera en Contabilidad y Auditoría, CPA, por la Pontificia Universidad Católica del Ecuador, Sede Santo Domingo, Ecuador

Institución: Pontificia Universidad Católica del Ecuador, Sede Santo Domingo, Ecuador

Dirección: Vía Chone Km 2, Santo Domingo, Ecuador, CP: 230203

E-mail: mlsalinaso@pucesd.edu.ec

\section{RESUMEN}

La mayor parte de las microempresas necesitan de un presupuesto de ventas para desenvolverse dentro de un mercado competitivo, por lo que la implementación es vital para su supervivencia a largo plazo. En este contexto el presente estudio tiene como objetivo determinar de qué manera la implementación de un presupuesto de ventas permitirá alcanzar la optimización de los ingresos de la empresa maderera "Don Junior" del cantón Puerto Quito, provincia de Pichicha, Ecuador. Para lo cual se llevó a cabo una investigación con enfoque mixto, de diseño no experimental longitudinal, de tipo descriptivo, exploratorio e histórico mediante encuestas aplicadas a los clientes fijos y entrevistas a los directivos generales. Los resultados reflejaron que el presupuesto de ventas debe ser implementado como una herramienta de gestión que le permita a la empresa un manejo adecuado de los gastos que vaya encaminado al crecimiento económico, ayudando a los directivos a la acertada toma de decisiones.

Palabras clave: presupuesto, ventas, optimización, gestión, gastos.

\begin{abstract}
Most micro-businesses need a sales budget to function in a competitive market, so its implementation is vital for long-term survival. In this context, the present study aims to determine how the implementation of a sales budget will allow to achieve the optimization of the income of the timber company "Don Junior" of the canton "Puerto Quito", Pichincha province, Ecuador. For which a research was carried out with a mixed approach, of a longitudinal non-experimental design, of a descriptive, exploratory and historical type, through surveys applied to fixed clients and interviews with general managers. The results reflected that the sales budget should be implemented as a management tool that allows the company to properly manage expenses that are aimed at economic growth, helping managers to make the right decisions.
\end{abstract}

Keywords: budget, sales, optimization, management, expenses. 


\section{INTRODUCCIÓN}

Dentro de las pequeñas y medianas empresas en Ecuador, los presupuestos de venta se han convertido en una metodología tradicional siendo fundamental para una correcta gestión empresarial debido a que el entorno donde se desarrollan es muy complejo y cambiante, por tal motivo se consideran a los presupuestos de venta como una herramienta administrativa alineada a un plan de acción de fácil interpretación que permite a las empresas saber cómo reaccionar ante cualquier variable interna o externa que repercuta de manera negativa en las ventas.

Según el autor López (2018) considera que los presupuestos de ventas se constituyen como una herramienta para la administración, gestión y control del departamento comercial y en general para el desarrollo de la empresa ya que brinda estimaciones de los niveles de ventas, ayuda a conocer la rentabilidad y sobre todo como se están manejando las operaciones empresariales internas mediante cronogramas para contemplar las estimaciones financieras, técnicas y administrativas que reflejen el potencial de ventas del negocio.

Un aporte fundamental que realizaron los autores Parra y Madriz (2017) sobre el manejo del presupuesto como instrumento de control financiero en pequeñas empresas de estructura familiar de la ciudad de Maracaibo-Venezuela, enfocado en identificar las debilidades con respecto a la utilización del presupuesto, determinar el uso del presupuesto como instrumento que contribuye al funcionamiento administrativo y precisar el control operativo dio como resultados que el presupuesto debe ser implementado como un programa que establezca bases para la sincronización de recursos, enfatizando en las metas económicas mediante la planificación y el control.

Otro estudio desarrollado por Luna et al. (2018) hace referencia a la implementación de una nueva técnica de análisis y estructuración en la construcción de un presupuesto empresarial en una microempresa del cantón Gualaceo Provincia del Azuay-Ecuador cuyo objetivo se enfocó en construir un presupuesto que permita capturar la dinámica económica de la empresa, optimizando los costos para lograr alcanzar las metas en el mediano y largo plazo, permitiendo determinar los niveles óptimos de presupuesto que puede alcanzar una empresa dado una estructura estimada de ingresos, ayudando a la alta gerencia direccionar variables y tomar las mejores decisiones.

Por otro lado, al momento de implementar un presupuesto de ventas es necesario realizar un análisis del comportamiento de muchas variables que tienen que ver con el mercado. Rincón \& Narváez (2017) manifiestan que existen varios métodos estadísticos y de planeación para presupuestar las ventas; entre ellos tenemos: 
- Método de estudio de mercadeo: se realiza un minucioso estudio de mercado, que plantea el posible resultado de los clientes ante algunas estrategias de publicidad planteadas.

- Método automático: se toman las ventas del año anterior, pues se espera que estas no varíen.

- Método promedio: se toman las diferencias resultantes de comparar las ventas de los últimos años, es decir la variación de un año a otro; estas diferencias se suman y se promedian según el número de años comparados.

- Método de aumentos o incremento porcentual: se toma las ventas de los últimos años, se calcula la variación porcentual de estos de un año a otro, estas variaciones porcentuales se suman y se promedian según el número de años tomados y el resultado se multiplica por las ventas del último año y luego se suma este nuevo dato a las ventas del último año.

La implementación de un presupuesto de ventas en la empresa maderera “Don Junior" servirá para medir el potencial interno de la organización. Siendo fundamental para la misma ya que permitirá ser una guía del volumen de ventas estimadas durante un determinado período. Además, ayudará a resolver problemas reales de gestión que tiene la empresa en cuanto al manejo de sus ventas y pretende beneficiar al propietario en el ámbito económico financiero, en busca de alcanzar la optimización adecuada de sus ingresos.

Por otra parte, existen normas que fundamentan esta investigación como las Normas NIIF para las PYMES (NIIF8, 2020) menciona que el uso de los presupuestos es fundamental en cualquier área especialmente en el área comercial ya que exigen de controles automáticos que permitan monitorear cualquier anomalía, que pueden ocurrir en diferentes períodos.

Esta investigación se encuentra relacionado con el objetivo 5 del Plan Nacional de Desarrollo Toda una Vida (SENPLADES, 2017-2021) que consiste en: "Impulsar la productividad para el crecimiento económico sostenible de manera redistributiva y solidaria", alineándose con la política 5.2 que se basa en promover la productividad, competitividad y calidad de los productos nacionales, como también la disponibilidad de servicios conexos y otros insumos, debido a que la investigación busca mejorar la gestión de las ventas de la empresa para alcanzar mayor productividad, encaminado a un adecuado manejo de los recursos y a su vez contribuir con el crecimiento y desarrollo a nivel de competitividad dentro del sector maderero.

Pico, Suárez y Tomalá (2017) mencionan que en las pequeñas y medianas empresas, se está aplicando la gestión presupuestaria, con el fin de tomar medidas que mejoren la situación de estas empresas, permitiendo al pequeño empresario prestar atención a la fijación de los objetivos financieros con base en los cuales planifican y controlan las diferentes actividades, logrando prever en forma inteligente tanto sus ingresos como gastos, por lo que la aplicación de un presupuesto de ventas debe 
iniciarse desde las empresas pequeñas con el objetivo de adelantarse al futuro y evitar déficit en la asignación de fondos monetarios, constituyendo hoy en día una de las claves del éxito de una empresa. Estos criterios concuerdan con lo expuesto por Valladares, et al. (2021) acerca del efecto de diversas variables de capital de trabajo en la rentabilidad de las pymes.

Por otro lado, de acuerdo con los datos obtenidos por el Instituto Nacional de Estadística y Censos (INEC, 2019) en Ecuador solo el 40\% de las empresas presentaron información detallada de sus ventas para el período 2018 de un total de 87.926 empresas que conforman el sector. Esta situación se debe a que gran parte de este sector está conformado por empresas pequeñas unipersonales o familiares cuyo flujo de ingresos es irregular, por lo que su participación en el mercado no es muy alta. Además, al analizar la estructura de ventas por tamaño de empresas se obtiene que para el año 2018 las empresas pequeñas alcanzaron un nivel de ventas equivalente al 6,26\% y las microempresas un nivel de ventas del 0,96\%".

Por lo que la falta de un presupuesto de ventas se ve reflejado principalmente en las microempresas que por lo regular manejan un nivel de ventas muy bajo ya que son muy susceptibles a los cambios del sector. Rincón \& Narváez (2017) mencionan que los presupuestos de venta son considerados como un parámetro inicial y base para los cálculos de otros presupuestos por lo que los datos que se obtengan son el norte de la empresa y sobre este se planifica todo el proceso productivo. Su estudio debe ser bastante riguroso y tomar referente de varias líneas de acción, para así evaluar el más cercano a una realidad.

El motivo principal por el que se plantea está propuesto de investigación es debido a que la empresa maderera "Don Junior" presentó un escenario de iliquidez y pérdidas consecutivas durante los períodos 2019 y 2020, los mismos que se encuentran reflejado en los respectivos balances contables de la empresa. Es importante considerar que se trata de una empresa pequeña y joven de origen familiar dentro de un sector amplio y competitivo como lo es el sector maderero, con flujos de ingresos irregulares en distintos períodos del año por lo que nunca ha contado con un presupuesto de ventas para su gestión

Según reportes de la empresa actualmente presenta ciertos problemas de gestión, por lo que, frente a la problemática descrita, el estudio tiene como motivo implementar un modelo de presupuesto para lograr que la empresa comience a generar mayores ingresos mejorando su gestión operativa. 


\section{MÉTODO}

\subsection{ENFOQUE, DISEÑO Y TIPO DE INVESTIGACIÓN}

En la presente investigación se aplicó un enfoque mixto, ya que se utilizó el enfoque cualitativo para la obtención de la información sobre la empresa en estudio mediante la entrevista, posteriormente se aplicó el enfoque cuantitativo debido a que se hizo uso de los estados financieros y reporte de la empresa para los años 2018 al 2020, así como la aplicación de técnicas y herramientas de análisis financiero.

El estudio se fundamenta en la metodología de investigación de Hernández, Fernández y Baptista (2014) que menciona que una investigación con enfoque mixto se caracteriza porque permite recolecta, analiza y establecer datos cuantitativos y cualitativos, en un mismo estudio, en la parte cuantitativa se centran en medir con mayor precisión la información obtenida de las encuestas en diferentes momentos para establecer con exactitud patrones de comportamiento en una población determinada y en la parte cualitativa permite la interpretación de las cosas en su contexto natural.

Esta investigación se enmarca en un diseño no experimental longitudinal, puesto que se realizó en base a conceptos y proceso ya establecidos, es decir, una investigación donde no se requiere la manipulación de las variables y la recolección de información se realizó en diferentes momentos del tiempo para analizar la evolución de las variables establecidas. De tipo descriptivo, exploratorio e histórico ya que se explicaron los procesos de gestión operativa y datos numéricos recogidos, analizando sus resultados y factores que inciden sobre los flujos de ingresos desde una visión general respecto a su realidad para lo cual se hizo uso de datos históricos sobre los niveles de ventas de períodos anteriores para su comparación.

\subsection{POBLACIÓN Y MUESTRA}

La presente investigación tiene como población finita a todos aquellos directivos generales de la empresa maderera "Don Junior" y clientes frecuentes de la ciudad de Guayaquil que emplean y comercialicen la madera como se muestra en la tabla 1.

Tabla 1. Población total de estudio

\begin{tabular}{lc}
\hline Población & Número \\
\hline Gerente General & 1 \\
Administrador & 1 \\
Clientes Fijos & 20 \\
Total & $\mathbf{2 2}$ \\
\hline Fuente: Empresa maderera "Don Junior"
\end{tabular}

En la presente investigación no hubo la necesidad de aplicar una muestra debido a que la población en estudio es pequeña de 22 personas siendo fácil de manejar. 


\subsection{TÉCNICAS DE RECOGIDA DE DATOS}

Se utilizó dos tipos de técnicas de recolección de datos, la primera se trata de la encuesta que se aplicó a los clientes fijos de la empresa maderera "Don Junior" mediante un cuestionario estructurado con preguntas cerradas con el fin de obtener información confiable que permita conocer la situación de las ventas del sector y poder determinar las variables externas más influyen para la elaboración del pronóstico de ventas. También se aplicó la entrevista a los directivos para conocer la gestión interna operativa y financiera en el desarrollo normal de sus operaciones mediante un cuestionario estructurado con preguntas abiertas.

\subsection{TÉCNICAS DE ANÁLISIS DE DATOS}

En la presente investigación la técnica de análisis de datos que se llevó a cabo para la parte cuantitativa fue mediante la tabulación de los datos a través de la herramienta de Microsoft Excel versión 2010, de igual manera se hizo uso de la estadística descriptiva y de los gráficos de frecuencias para referirse a las respuestas de la encuesta y para la parte cualitativa se analizó mediante comparaciones entre años anteriores con el fin de analizar el manejo interno y externo de la empresa en cuanto a la gestión de las ventas.

\section{RESULTADOS}

\subsection{RESULTADO DE LA ENTREVISTA DIRIGIDA AL GERENTE DE LA EMPRESA}

La presente entrevista fue realizada al Gerente propietario de la empresa maderera "Don Junior" el Sr. Francisco Javier Salinas Baldeón, en la cual se establecieron ciertas preguntas con respecto al giro del negocio. La información obtenida permitió reflejar ciertas falencias en la organización, donde se encontró que la empresa en años anteriores no ha contado con un presupuesto de ventas y que se ve en la necesidad de aplicarlo por el motivo de que las ventas del negocio se han visto afectadas considerablemente, aparte se está teniendo un exceso en los gastos operativos, por no existir una clasificación adecuada de aquellos gastos afines al negocio, especialmente en los gastos de viáticos y varios ocasionando un desfase para la empresa.

La falta de capital, está provocando que no se aproveche al máximo la capacidad productiva de la empresa y por ende el nivel de ventas no es muy alto, el mismo que es aprovechado por la competencia las cuales son la empresa maderera "Ana Victoria", "Jean Ambuludí" y "El Colorado" que están abarcando esa parte de la demanda que la empresa podría cubrir. 


\subsection{RESULTADO DE LA ENTREVISTA DIRIGIDA AL ADMINISTRADOR DE LA EMPRESA.}

En la entrevista realizada al administrador de la empresa maderera "Don Junior", el Sr. Welinton Javier Salinas Ordoñez se realizaron varias preguntas con respecto al manejo operativo. Se pudo identificar ciertas cuestiones importantes que se van a recalcar a continuación: la empresa no realiza una revisión frecuente de los estados e informes financieros por lo que cualquier irregularidad no se puede verificar hasta el final del período. Por otro lado, un factor importante que no está considerando la empresa al momento de producir es la estacionalidad que tiene las ventas en este sector.

Por lo general entre los meses de junio a noviembre existe un leve incremento en las ventas, en cambio para los meses de diciembre a mayo se establece un decremento considerable de las mismas por la dificulta en el traslado de la madera debido a las lluvias. Además, no todos los inventarios rotan con la misma frecuencia a pesar de que la mayor parte de las ventas se realizan de manera semanal normalmente es para un solo tipo de madera.

\subsection{RESULTADOS DE LA ENCUESTA DIRIGIDA A LOS CLIENTES FIJOS DE LA EMPRESA MADERERA "DON JUNIOR"}

La presente encuesta fue aplicada a 20 clientes fijos de la ciudad de Guayaquil que emplean y comercializan la madera, con el fin de conocer las variables externas que puedan influir de manera directa en las ventas dentro de los próximos períodos, resultados que se ven reflejados en las figuras 1 al 4.

Figura 1. Resultados de las preguntas 1, 2 y 3 sobre la frecuencia de los pedidos, mayor nivel de ventas y tipo de madera que se adquiere.

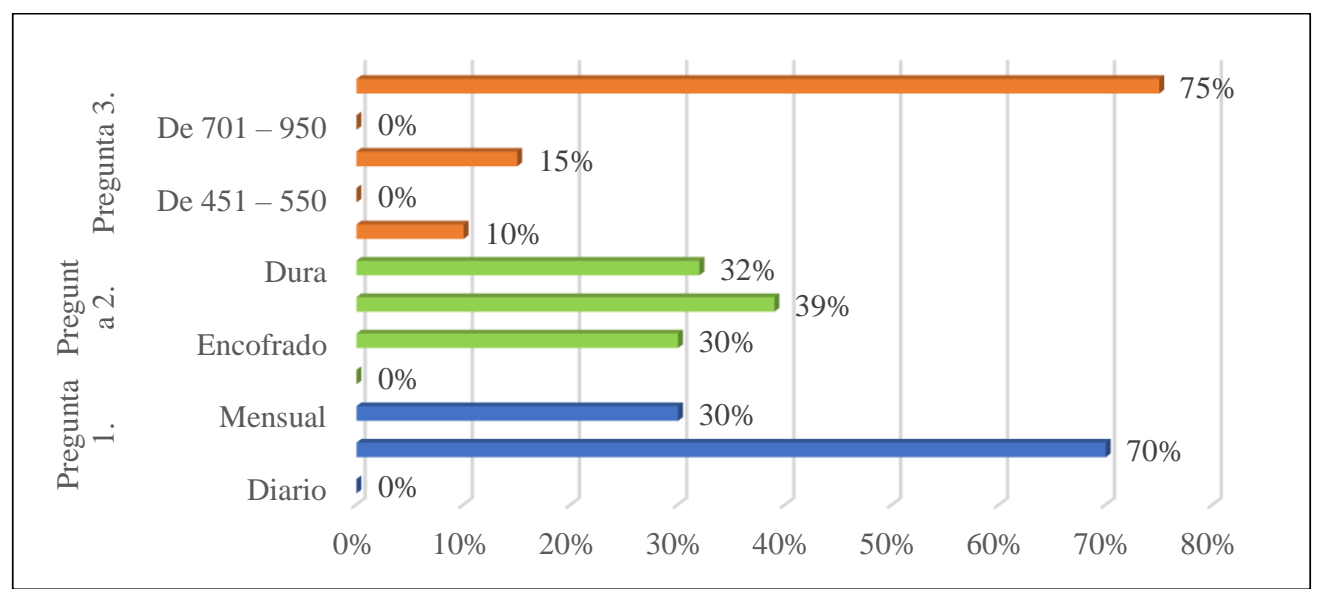

Fuente: Encuesta realizada a los clientes de la empresa maderera "Don Junior".

Se pudo identificar que el $70 \%$ de los clientes realizan los pedidos de manera semanal, por otro lado, la madera que tiene un nivel de ventas mayor es la semidura que representa el $39 \%$ de las ventas globales, esto debido a que este tipo de madera es la más utilizada para construcciones, ya que la madera de encofrado es muy delicada y la madera dura es muy pesada y por ende no se puede clavar en ella, 
También el 75\% de los clientes encargan a la empresa pedidos desde 951 tablones de madera en adelante que representa una cantidad considerable de ingresos para la misma. Sin embargo, el problema radica que la empresa produce cantidades similares para los tres tipos de madera generando que muchas veces el inventario de la madera dura y de encofrado no salga rápido a pesar de que los pedidos se realizan de manera semanal el 39\% solo son para la madera semidura que son las que tienen los pedidos más altos.

Figura 2. Resultados de las preguntas 4, 5 y 6 sobre los aspectos que se toman en cuenta para la compra, la variación del precio y la cantidad que suele variar.

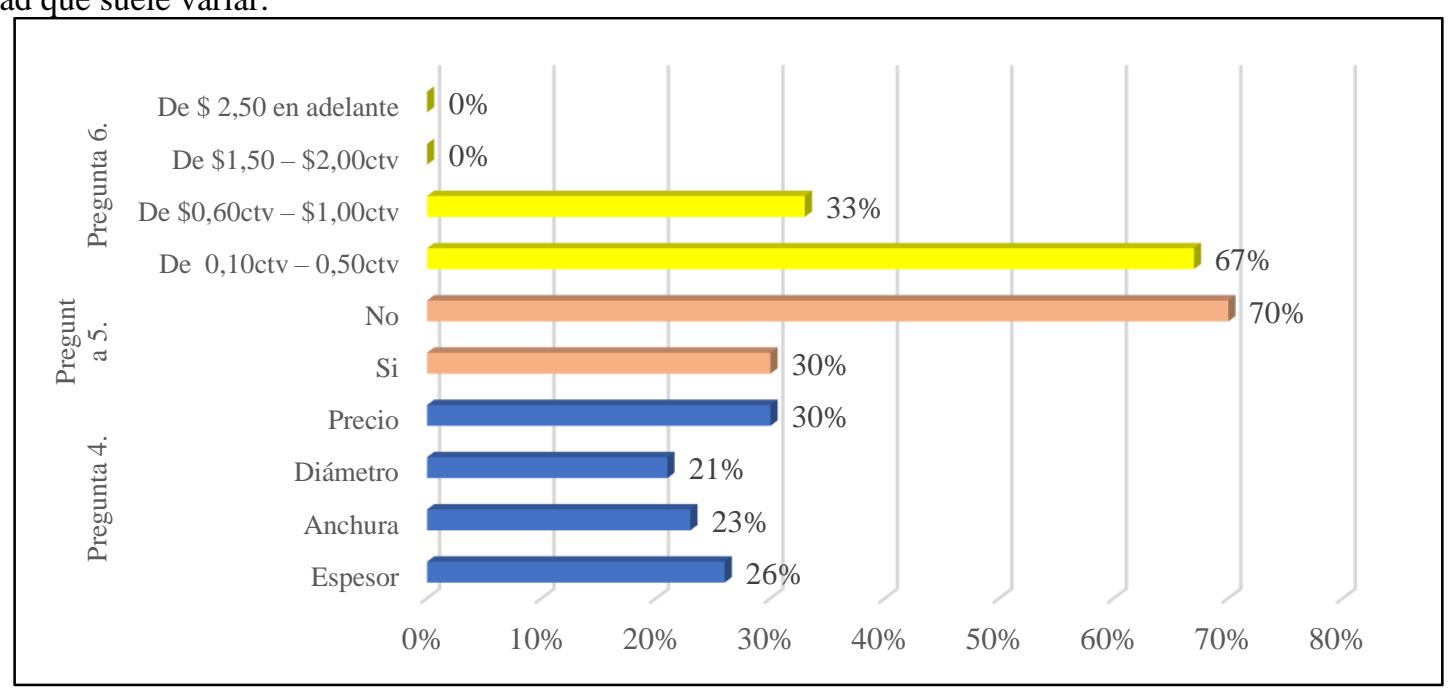

Fuente: Encuesta realizada a los clientes de la empresa maderera "Don Junior".

Se pudo determinar que el 30\% de los clientes consideran como factor principal en la compra el precio, por otro lado, el $70 \%$ de los clientes consideran que el precio de la madera no varía con frecuencia en el mes. Mientras que $30 \%$ de ellos consideran que si existe una variación y es normalmente entre 0.10 ctv. a 0.50 ctv., aspecto que es favorable para la empresa ya que una parte de los clientes consideran como factor principal el precio para ejecutar su compra y al existir un $70 \%$ que consideran que no existe una variación se podría decir que sector maderero es estable en cuanto a precios del mercado. 
Figura 3. Resultados de las preguntas 7, 8 y 9 sobre las políticas de venta de la empresa, calidad en el tiempo de entrega e inconvenientes con la calidad del producto.

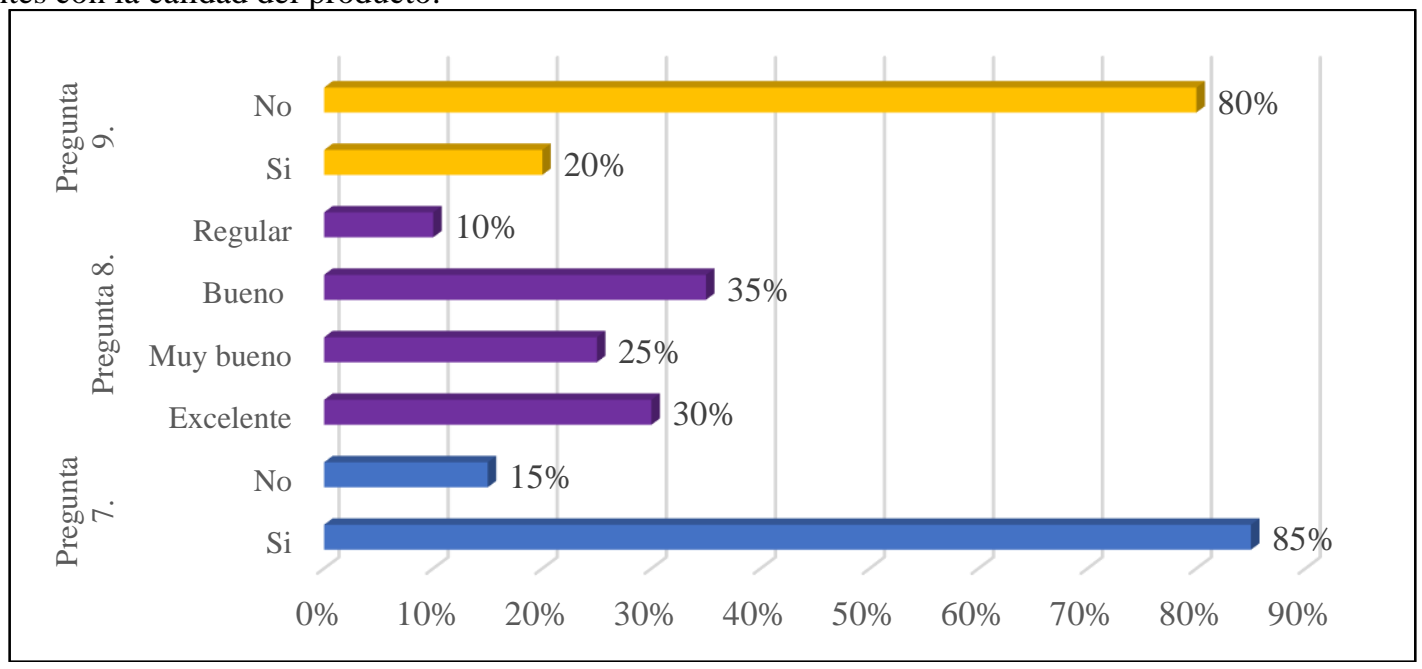

Fuente: Encuesta realizada a los clientes de la empresa maderera "Don Junior".

Se determinó que el $85 \%$ de los clientes si conocen con claridad las políticas de venta que maneja la empresa por lo que existe una comunicación adecuada entre cliente-empresa. De igual forma el $80 \%$ de ellos manifestaron que no han existido inconvenientes con el estado del producto requerido y que la calidad en el tiempo de entrega de la madera a su lugar de destino es buena. El hecho de que una parte de los clientes no consideren que el tiempo de entrega de la madera es excelente, se debe que en ciertas circunstancias la empresa ha tenido inconvenientes de retraso de la madera por factores inesperados externos y problemas con la guía de movilización por cambio de especie.

Figura 4. Resultados de las preguntas 10, 11 y 12 sobre inconvenientes con la calidad del producto, la atención al cliente y la perspectiva de los clientes con respecto a los controles de venta.

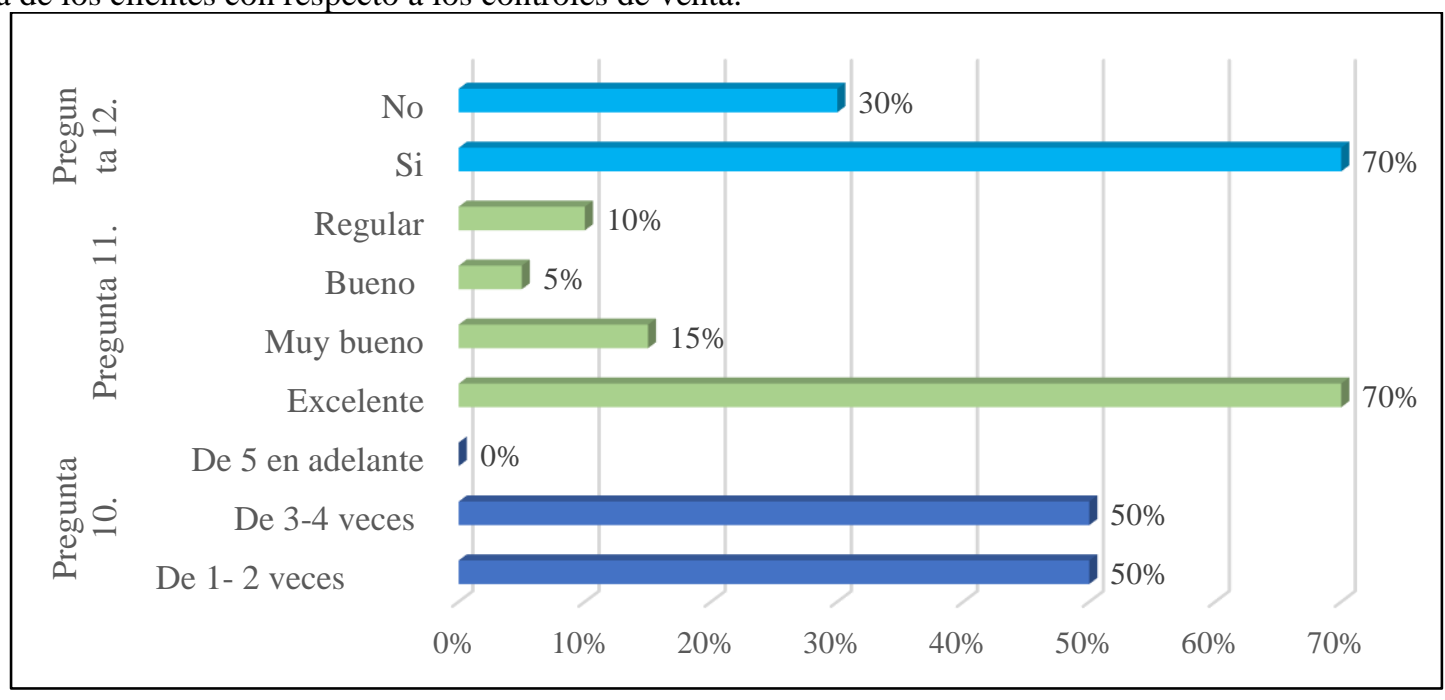

Fuente: Encuesta realizada a los clientes de la empresa maderera "Don Junior". 
Se pudo determinar que el 50\% de los clientes han tenido inconvenientes entre 1 a 4 veces en algún momento que representan 4 clientes de los 20 encuestados.

Por otro lado, el $70 \%$ de ellos manifestaron que la atención al cliente que brinda la empresa es excelente y tan solo el $10 \%$ indicó que es regular por lo que se entiende que a pesar de pequeños inconvenientes que ha tenido con algunos clientes la empresa ha sabido solucionar de la mejor manera conservando la fidelidad de los mismo que es un factor importante para la continuidad de la empresa y la sostenibilidad e incremento de las ventas.

\subsection{RESULTADOS DEL ANÁLISIS COMPARATIVO DE LAS VENTAS}

Tabla 2. Análisis comparativo de las ventas para el período 2018 - 2020.

\begin{tabular}{lccccc}
\hline & \multicolumn{1}{c}{ VENTAS ANUALES POR MADERA } & & VARIACIÓN \\
\hline INFORME & $\mathbf{2 0 1 8}$ & $\mathbf{2 0 1 9}$ & $\mathbf{2 0 2 0}$ & $\mathbf{2 0 1 9}$ & $\mathbf{2 0 2 0}$ \\
\hline Ventas de madera encofrado & $\$ 124,800.00$ & $\$ 122,148.00$ & $\$ 125,307.00$ & $-2 \%$ & $3 \%$ \\
Ventas de madera semidura & $\$ 192,008.10$ & $\$ 151,632.00$ & $\$ 146,208.00$ & $-27 \%$ & $-4 \%$ \\
Ventas de madera dura & $\$ 159,120.00$ & $\$ 147,370.00$ & $\$ 132,700.00$ & $-8 \%$ & $-11 \%$ \\
\hline INFORME & INGRESOS ANUALES & $\%$ & & \\
Ventas totales 2018 & $\$ 475,928.10$ & & & \\
Ventas totales 2019 & $\$ 421,150.00$ & $-13 \%$ & & & \\
Ventas totales 2020 & $\$ 404,215.00$ & $-4 \%$ & & &
\end{tabular}

Fuente: Informes de venta de la empresa maderera "Don Junior".

El nivel de ventas para el año 2019 decreció para los tres tipos de madera con un $2 \%$ para la madera encofrado, un $27 \%$ para la semidura y un $8 \%$ para la dura dando una variación negativa del $13 \%$ con respecto al año 2018, esto debido a que el mercado interno tuvo una tasa promedio de variación interanual del -2,0\% que repercutió fuertemente en las empresas del sector. Para el año 2020 la situación no cambio tanto debido a la situación de la pandemia, las ventas para la madera semidura y dura continuaron con una disminución del 4\% y $11 \%$ respectivamente al año anterior, sin embargo la madera encofrado aumentó un $3 \%$ teniendo una variación global negativa de ventas del $4 \%$.

\subsection{RESULTADOS DE LOS DESEMBOLSOS DE LA EMPRESA}

Tabla 3. Análisis de los desembolsos de la empresa para los años $2018-2020$.

\begin{tabular}{|c|c|c|c|c|c|c|c|}
\hline \multirow[b]{2}{*}{ GASTOS DE VENTA } & \multicolumn{3}{|c|}{ GASTOS OPERACIONALES } & \multicolumn{2}{|c|}{ VARIACIÓN \$ } & \multicolumn{2}{|c|}{ VARIACIÓN \% } \\
\hline & 2018 & 2019 & 2020 & $2018 / 2019$ & $\begin{array}{c}2019 / 202 \\
0 \\
\end{array}$ & $\begin{array}{c}2018 / 201 \\
9 \\
\end{array}$ & $\begin{array}{c}2019 / 202 \\
0 \\
\end{array}$ \\
\hline Transporte & $\$ 48,000.00$ & $\$ 51,120.00$ & $\$ 64,800.00$ & $3,120.00$ & $13,680.00$ & $6 \%$ & $21 \%$ \\
\hline
\end{tabular}




\begin{tabular}{|c|c|c|c|c|c|c|c|}
\hline Obreros & $\$ 8,400.00$ & $\$ 9,120.00$ & $\$ 7,200.00$ & 720.00 & $-1,920.00$ & $8 \%$ & $-27 \%$ \\
\hline Gabarra & $\$ 30,000.00$ & $\$ 34,920.00$ & $\$ 34,800.00$ & $4,920.00$ & -120.00 & $14 \%$ & $-0.3 \%$ \\
\hline Guía de Movilización & $\$ 68,400.00$ & $\$ 72,000.00$ & $\$ 74,400.00$ & $3,600.00$ & $2,400.00$ & $5 \%$ & $3 \%$ \\
\hline Viáticos & $\$ 48,000.00$ & $\$ 61,680.00$ & $\$ 57,600.00$ & $13,680.00$ & $-4,080.00$ & $22 \%$ & $-7 \%$ \\
\hline Gastos varios & $\$ 33,720.00$ & $\$ 62,760.00$ & $\$ 48,960.00$ & $29,040.00$ & $\begin{array}{c}- \\
13,800.00\end{array}$ & $46 \%$ & $-28 \%$ \\
\hline $\begin{array}{l}\text { GASTOS } \\
\text { ADMINISTRATIVOS }\end{array}$ & 2018 & 2019 & 2020 & $2018 / 2019$ & $\begin{array}{c}2019 / 202 \\
0 \\
\end{array}$ & $\begin{array}{c}2018 / 201 \\
9 \\
\end{array}$ & $\begin{array}{c}2019 / 202 \\
0 \\
\end{array}$ \\
\hline Administrador & $\$ 10,200.00$ & $\$ 12,000.00$ & $\$ 12,000.00$ & $\$ 1,800.00$ & 0.00 & $15 \%$ & $0 \%$ \\
\hline Contadora & $\$ 8,400.00$ & $\$ 9,000.00$ & $\$ 9,000.00$ & $\$ 600.00$ & 0.00 & $7 \%$ & $0 \%$ \\
\hline $\begin{array}{l}\text { TOTAL GASTOS } \\
\text { OPERATIVOS } \\
\end{array}$ & $\begin{array}{c}\$ \\
255,120.00 \\
\end{array}$ & $\begin{array}{c}\$ \\
312,600.00 \\
\end{array}$ & $\begin{array}{c}\$ \\
308,760.00\end{array}$ & $\begin{array}{c}\$ \\
\mathbf{5 7 , 4 8 0 . 0 0} \\
\end{array}$ & $\begin{array}{c}-\$ \\
3,840.00 \\
\end{array}$ & $18 \%$ & $-1.2 \%$ \\
\hline
\end{tabular}

Fuente: Informes de gastos de la empresa maderera "Don Junior".

El 2019 fue el año que la empresa incurrió una mayor cantidad en gastos operacionales especialmente en la cuenta de viáticos y gastos varios que tuvieron un incremento del $22 \%$ y $46 \%$ respectivamente desembolsando un total de $\$ 57.480 .00$ dólares más que el 2018 , incremento que no se encontró debidamente justificado por la empresa ya que en los registros analizados muchos valores no estaban respaldados con facturas y otros valores no tenían relación con el giro del negocio.

Siendo gastos personales del propietario, situación que es preocupante para la empresa ya que el nivel de ventas es inferior para poder cubrir dichos desembolsos. Mientras que para el año 2020 a pesar de que el gasto fue menor en $1.23 \%$ el valor siguió siendo alto para la empresa, lo que generó que existiera una pérdida considerable para aquel período.

\subsection{RESULTADOS DEL ANÁLISIS DEL PRONÓSTICO DE VENTAS EMPRESARIAL}

Tabla 4. Pronóstico estacional en unidades para los tres tipos de madera.

\begin{tabular}{|c|c|c|c|c|c|}
\hline \multicolumn{6}{|c|}{ PRONÓSTICO DE UNIDADES PARA LA MADERA ENCOFRADO } \\
\hline PERÍODO & AÑO & MESES & PRONÓSTICO & ÍNDICE ESTACIONAL & P. ESTACIONAL CON TENDENCIA \\
\hline 37 & \multirow{12}{*}{2021} & Enero & $4,591.96$ & 0.89 & 4078 \\
\hline 38 & & Febrero & $4,604.37$ & 0.87 & 3985 \\
\hline 39 & & Marzo & $4,616.82$ & 0.88 & 4044 \\
\hline 40 & & Abril & $4,629.30$ & 0.79 & 3667 \\
\hline 41 & & Mayo & $4,641.82$ & 1.03 & 4785 \\
\hline 42 & & Junio & $4,654.37$ & 1.04 & 4844 \\
\hline 43 & & Julio & $4,666.95$ & 1.07 & 4995 \\
\hline 44 & & Agosto & $4,679.57$ & 1.11 & 5216 \\
\hline 45 & & Septiembre & $4,692.22$ & 1.14 & 5362 \\
\hline 46 & & Octubre & $4,704.91$ & 1.16 & 5452 \\
\hline 47 & & Noviembre & $4,717.63$ & 1.09 & 5126 \\
\hline 48 & & Diciembre & $4,730.39$ & 0.93 & 4417 \\
\hline
\end{tabular}




\begin{tabular}{|c|c|c|c|c|c|}
\hline 37 & \multirow{10}{*}{2021} & Enero & $3,736.28$ & 0.90 & 3367 \\
\hline 38 & & Febrero & $3,718.02$ & 0.83 & 3092 \\
\hline $\begin{array}{l}39 \\
40\end{array}$ & & $\begin{array}{l}\text { Marzo } \\
\text { Abril }\end{array}$ & $\begin{array}{l}3,699.85 \\
3,681.77\end{array}$ & $\begin{array}{l}0.69 \\
0.51\end{array}$ & $\begin{array}{l}2569 \\
1891\end{array}$ \\
\hline 41 & & Mayo & $3,663.78$ & 1.04 & 3802 \\
\hline $\begin{array}{l}42 \\
43\end{array}$ & & $\begin{array}{l}\text { Junio } \\
\text { Julio }\end{array}$ & $\begin{array}{l}3,645.88 \\
3,628.06\end{array}$ & $\begin{array}{l}1.09 \\
1.14\end{array}$ & $\begin{array}{l}3968 \\
4118\end{array}$ \\
\hline 44 & & Agosto & $3,610.33$ & 1.20 & 4334 \\
\hline 45 & & Septiembre & $3,592.69$ & 1.21 & 4341 \\
\hline 46 & & Octubre & $3,575.14$ & 1.21 & 4312 \\
\hline 47 & & Noviembre & $3,557.67$ & 1.15 & 4097 \\
\hline 48 & & Diciembre & $3,540.28$ & 1.03 & 3651 \\
\hline \multicolumn{6}{|c|}{ PRONÓSTICO DE UNIDADES PARA LA MADERA DURA } \\
\hline 37 & \multirow{12}{*}{2021} & Enero & $2,356.19$ & 0.88 & 2083 \\
\hline 38 & & Febrero & $2,351.11$ & 0.80 & 1893 \\
\hline 39 & & Marzo & $2,346.04$ & 0.68 & 1594 \\
\hline 40 & & Abril & $2,340.98$ & 0.61 & 1433 \\
\hline 41 & & Mayo & $2,335.93$ & 1.06 & 2481 \\
\hline 42 & & Junio & $2,330.90$ & 1.10 & 2562 \\
\hline 43 & & Julio & $2,325.87$ & 1.15 & 2676 \\
\hline 44 & & Agosto & $2,320.86$ & 1.15 & 2664 \\
\hline 45 & & Septiembre & $2,315.86$ & 1.15 & 2656 \\
\hline 46 & & Octubre & $2,310.86$ & 1.17 & 2712 \\
\hline 47 & & Noviembre & $2,305.88$ & 1.14 & 2635 \\
\hline 48 & & Diciembre & $2,300.91$ & 1.10 & 2520 \\
\hline
\end{tabular}

Fuente: Informes de venta de la empresa maderera "Don Junior".

Se realizó el pronóstico de ventas considerando la demanda individual para cada tipo de madera, tomando en consideración el modelo de regresión logarítmica con tendencia estacional mediante la utilización de la herramienta de Excel 2010. De acuerdo con el autor Moreno (2019) menciona que los modelos de regresión son técnicas estadísticas que utilizan información histórica para establecer pronósticos futuros, factible cuando la demanda o ventas tienen un patrón continuo en común.

Al analizar los niveles de ventas para los años 2018 al 2020 reflejó una tendencia con estacionalidad durante los tres años en donde las ventas crecieron a partir del mes de junio hasta noviembre, especialmente de septiembre a noviembre y decayeron para los meses de diciembre a mayo debido a la temporada invernal, razón por la cual se siguió ese patrón. Se calculó el índice estacional y se multiplicó con la demanda real para obtener la demanda estacional y también se consideró la estacionalidad del sector. 


\subsection{RESULTADOS DEL PRESUPUESTO DE VENTAS Y SU IMPACTO EN LA OPTIMIZACIÓN DE INGRESOS Y PROCESO DE TOMA DE DECISIONES.}

Tabla 5. Presupuesto de ventas para la madera encofrado de la Empresa Maderera "Don Junior". Del 01 de Enero al 31 de Diciembre del 2021

\begin{tabular}{lccc}
\hline PERÍODO & UNIDADES & PRECIO & TOTAL VENTAS \\
\hline Enero & 4,282 & $\$ 2.31$ & $\$ 9,891.99$ \\
Febrero & 4,184 & $\$ 2.31$ & $\$ 9,664.41$ \\
Marzo & 4,247 & $\$ 2.31$ & $\$ 9,809.55$ \\
Abril & 3,850 & $\$ 2.31$ & $\$ 8,894.23$ \\
Mayo & 5,024 & $\$ 2.75$ & $\$ 13,816.84$ \\
Junio & 5,086 & $\$ 2.75$ & $\$ 13,985.80$ \\
Julio & 5,245 & $\$ 2.75$ & $\$ 14,423.59$ \\
Agosto & 5,477 & $\$ 2.75$ & $\$ 15,062.63$ \\
Septiembre & 5,630 & $\$ 2.75$ & $\$ 15,482.87$ \\
Octubre & 5,724 & $\$ 2.75$ & $\$ 15,741.30$ \\
Noviembre & 5,383 & $\$ 2.75$ & $\$ 14,802.54$ \\
Diciembre & 4,638 & $\$ 2.31$ & $\$ 10,712.77$ \\
\hline VENTAS ANUAL & & $\$ 152,288.52$ \\
\hline
\end{tabular}

Fuente: Informes de venta de la empresa maderera "Don Junior".

Tabla 6. Presupuesto de ventas para la madera semidura de la Empresa Maderera "Don Junior". Del 01 de Enero al 31 de Diciembre del 2021

\begin{tabular}{lccc}
\hline PERÍODO & UNIDADES & PRECIO & TOTAL VENTAS \\
\hline Enero & 3,872 & $\$ 3.30$ & $\$ 12,776.95$ \\
Febrero & 3,556 & $\$ 3.30$ & $\$ 11,735.88$ \\
Marzo & 2,954 & $\$ 3.30$ & $\$ 9,749.45$ \\
Abril & 2,175 & $\$ 3.30$ & $\$ 7,176.07$ \\
Mayo & 4,372 & $\$ 3.63$ & $\$ 15,870.10$ \\
Junio & 4,563 & $\$ 3.63$ & $\$ 16,564.37$ \\
Julio & 4,736 & $\$ 3.63$ & $\$ 17,192.39$ \\
Agosto & 4,984 & $\$ 3.63$ & $\$ 18,093.72$ \\
Septiembre & 4,993 & $\$ 3.63$ & $\$ 18,123.49$ \\
Octubre & 4,959 & $\$ 3.63$ & $\$ 18,001.16$ \\
Noviembre & 4,711 & $\$ 3.63$ & $\$ 17,102.12$ \\
Diciembre & 4,199 & $\$ 3.30$ & $\$ 13,857.18$ \\
\hline VENTAS ANUAL & $\mathbf{5 0 . 0 7 5}$ & $\$ \mathbf{1 7 6 , 2 4 2 . 8 7}$ \\
\hline
\end{tabular}

Fuente: Informes de venta de la empresa maderera "Don Junior".

Tabla 7. Presupuesto de ventas para la madera dura de la Empresa Maderera “Don Junior". Del 01 de Enero al 31 de Diciembre del 2021

\begin{tabular}{lccc}
\hline PERÍODO & UNIDADES & PRECIO & TOTAL VENTAS \\
\hline Enero & 2,295 & $\$ 4.95$ & $\$ 11,361.31$ \\
Febrero & 2,085 & $\$ 4.95$ & $\$ 10,322.44$ \\
Marzo & 1,757 & $\$ 4.95$ & $\$ 8,696.77$
\end{tabular}




\begin{tabular}{llll} 
Abril & 1,580 & $\$ 4.95$ & $\$ 7,819.30$ \\
Mayo & 2,735 & $\$ 5.50$ & $\$ 15,041.87$ \\
Junio & 2,740 & $\$ 5.50$ & $\$ 15,068.49$ \\
Julio & 2,883 & $\$ 5.50$ & $\$ 15,854.14$ \\
Agosto & 3,018 & $\$ 5.50$ & $\$ 16,601.20$ \\
Septiembre & 2,995 & $\$ 5.50$ & $\$ 16,474.12$ \\
Octubre & 2,991 & $\$ 5.50$ & $\$ 16,449.21$ \\
Noviembre & 2,906 & $\$ 5.50$ & $\$ 15,983.28$ \\
Diciembre & 2,779 & $\$ 4.95$ & $\$ 13,756.79$ \\
\hline VENTAS ANUAL & $\mathbf{3 0 . 7 6 4}$ & $\$ \mathbf{1 6 3 , 4 2 8 . 9 1}$
\end{tabular}

Fuente: Informes de venta de la empresa maderera "Don Junior”.

Se presupuestó un nivel de ventas anual para la madera de encofrado de \$152.288.52 dólares, y se deberá producir 58.770 unidades para alcanzar ese nivel de ventas, por otro lado, para la demanda semidura se determinó un nivel de ventas de \$176.242.87 dólares produciendo 50.075 unidades y para la madera dura un nivel de ventas de \$163.428.91 dólares produciendo 30.764 unidades, dando un total de ventas para el período 2021 de $\$ 491.960$ dólares.

Tabla 8. Cuadro de optimización de los ingresos, costos y gastos.

\begin{tabular}{lcccc}
\hline DATOS & $\mathbf{2 0 1 9}$ & $\mathbf{2 0 2 0}$ & $\mathbf{2 0 2 1}$ & OPTIMIZACIÓN \\
\hline Ingresos & $\$ 421,200.00$ & $\$ 404,215.00$ & $\$ 491,960.30$ & $17.84 \%$ \\
Costos & $\$ 270,000.00$ & $\$ 229,500.00$ & $\$ 206,623.33$ & $11.07 \%$ \\
Gastos & $\$ 312,600.00$ & $\$ 308,760.00$ & $\$ 253,674.00$ & $21.72 \%$ \\
Utilidad / pérdida & $-\$ 161,400.00$ & $-\$ 134,045.00$ & $\$ 31,662.97$ & \\
\hline \multicolumn{5}{c}{ Fuente: Informes de venta de la empresa maderera "Don Junior". }
\end{tabular}

La empresa alcanzando los niveles de ventas presupuestadas por madera y considerando una reducción en los gastos operativos especialmente en las cuentas de viáticos y gastos varios mediante una clasificación adecuada de los gastos solo del negocio, tomando en cuenta documentación histórica se ha estipulado un nivel de gastos disponibles para el período de \$253.674.00 dólares, presupuesto acorde con las unidades proyectadas, generando una optimización de ingresos del $17.84 \%$, de costos del $11.07 \%$ y sobre todo una reducción del $21.72 \%$ de los gastos del período permitiendo obtener una utilidad marginal de \$31.662.97 dólares.

\section{DISCUSIÓN}

En el presenta trabajo de investigación se procedió a realizar el levantamiento de información para determinar la situación actual de la gestión de las ventas de la empresa con el fin de utilizar la información para la implementación del presupuesto de ventas, que permitió enfatizar las falencias que tienen la entidad que se describen a continuación. 
Existe una baja rotación de inventarios para la madera dura y encofrado, ya que, la empresa produce cantidades similares para los tres tipos de madera, lo que hace que la madera dura y encofrado salga, pero se demore más tiempo en venderse. También la falta de capital de trabajo está provocando que la empresa no pueda producir con su capacidad máxima, sobreestimación de los gastos de venta, y en general una gestión deficiente de las ventas que se agravan al no considerar la estacionalidad del sector al momento de producir.

Por lo que de acuerdo con el autor Rivadeneira (2018) menciona que es importante que la empresa tenga conocimiento sobre la rapidez con que venden los productos para la toma de decisiones de esa forma se puede cambiar el volúmen de fabricación, ajustar el precio del producto o poder establecer instrumentos de gestión para mejorar la rotación y uno de esos instrumentos que se puede utilizar es el presupuesto de ventas que va ayudar a la empresa a tener un control de sus inventarios, optimizando recursos, tiempo y siendo más productivos.

Por otro lado el índice de deserción de los clientes reflejó que la empresa para el año 2019 perdió el $13 \%$ de sus clientes, situación que se vio reflejada en las ventas ya que existió una disminución para aquel periodo, debido también a ciertos inconvenientes en la entrega de los pedidos, sin embargo al comparar con el periodo actual la empresa ha mejorado ya que de acuerdo con las encuestas realizadas a los clientes el $80 \%$ no ha tenido inconvenientes con la entrega del producto y tampoco hubo una deserción de clientes, demostrando que la empresa ha sabido mantener la fidelidad de sus clientes, factor que es fundamental para el crecimiento de la misma, ya que al ser una empresa pequeña el impacto es mayor al perder clientes.

Con respecto al índice de rendimiento de producción la empresa desde el año 2019 ya venía teniendo problemas en cumplir con la producción planificada, factor que se vio reflejado para el periodo 2020 con un porcentaje del 11\% de producción insatisfecha y para el periodo actual sigue siendo un problema por la falta de capital de trabajo disponible aunque en menor grado debido a las acciones impulsadas por el gerente de la empresa para mejorar el capital disponible y producir con su capacidad normal.

Para identificar la variación de las ventas se hizo uso de los Estados de Resultados de la empresa para los años 2018 al 2020 donde se evidenció que para el año 2019 no hubo un índice de crecimiento de las ventas más bien disminuyó un $13 \%$ producto de varios factores entre ellos que el índice de participación del mercado de la empresa fue del 6\% considerando sólo el área donde está situada la entidad que es el cantón Puerto Quito por lo cual se tomó en cuenta la participación de ventas para la provincia de Pichincha. 
Otro de los factores sería el no considerar la estacionalidad de las ventas que según reportes del Banco Central del Ecuador (2019) el sector maderero tuvo una tasa promedio de variación interanual de $-2,0 \%$ entre 2006 y 2019. En cuanto al año 2020 las ventas disminuyeron un 4\%, situación que no fue tan desfavorable para la empresa considerando que la pandemia del Covid-19 estaba en su mayor auge principalmente en los primeros meses del año, sin embargo, el sector maderero logró recuperarse de una manera histórica en el último trimestre del año, permitiendo que la empresa tuviera una variación baja en las ventas.

En cuanto al impacto de los costos y gastos se pudo comprobar que los gastos de venta para el año 2019 aumentaron un 18\%, mientras que para el año 2020 hubo una disminución del $1.23 \%$, siendo todavía un valor alto ya que no se pudo compensar el total de desembolsos con el nivel de ventas generado, provocando una pérdida de $\$ 88,871.84$, que representó una pérdida menor del $17 \%$ con respecto al 2019 , y conforme a la entrevista realizada al gerente se determinó que la empresa registra los gastos de manera empírica por lo que no existe clasificación adecuada incluyendo también otros gastos no relacionados con el giro del negocio, lo que sigue provocando que exista una sobreestimación de los mismos.

Por último, todas estas falencias detectadas en el proceso de levantamiento de información van a servir como base para buscar el mejoramiento en la gestión de las ventas de la empresa a través de la implementación de un presupuesto de ventas de acuerdo con la realidad tanto interna como externa, ya que, de acuerdo con los autores Parra y Madriz (2017) en la investigación que realizaron sobre manejo del presupuesto en pequeñas empresas de estructura familiar de la ciudad de Maracaibo-Venezuela, mencionan que, por medio de un presupuesto de ventas permite solucionar debilidades de gestión, financieras y administrativas, problemas que se puedo evidenciar en el presente trabajo.

De igual manera, otro estudio desarrollado por Luna et al. (2018) para la implementación de un presupuesto empresarial en las microempresas, mencionan que es fundamental su realizacion en estas empresas, ya que, al ser pequeñas la mayor parte no tienen una estructura eficiente en la gestión las ventas y por medio del presupuesto empresarial se va poder sincronizar los recursos, haciendo énfasis en las metas económicas.

\section{CONCLUSIONES}

En el proceso de levantamiento de información mediante los instrumentos de recogida de datos que se utilizó se concluye que la empresa registra de manera empírica los costos y gastos de venta hasta el punto de comercialización, por lo que, no cuenta con una estructura de clasificación adecuada de los mismos, incluyendo también otros gastos no relacionados con el giro del negocio provocando una 
sobrestimación de lo presupuestado especialmente en los rubros de viáticos y gastos varios que no se encuentran debidamente justificados por la empresa.

Una vez analizado los Estados de Resultados de la empresa para los períodos 2018 al 2020 se puedo determinar que el nivel de ventas para el año 2018 tuvo una caída del 13\% debido a que la madera semidura tuvo un descenso del $27 \%$ que es la que generó mayores ingresos para el año 2018. En el año 2020 el nivel de ventas disminuyó en un 4\% más debido a la paralización de las actividades a causa del Covid-19. A pesar de eso la variación de las ventas fue baja y actualmente el nivel de ventas de la empresa es estable, aspecto que se comprobó con la entrevista al gerente y las encuestas a los clientes con referencia al nivel y frecuencia de pedidos realizados.

En cuanto a las variables internas que están influenciando en la deficiencia de la gestión de las ventas está la baja rotación de inventarios especialmente para la madera dura y encontrado debido a que la empresa no produce de acuerdo a la demanda estacional de cada tipo de madera y otro de los problemas es la falta de capital de trabajo disponible por lo que el gerente está buscando nuevas fuentes de financiamiento que permitan a la empresa aprovechar su capacidad productiva.

De acuerdo con la entrevista realizada al administrador se pudo determinar que la empresa al momento de producir no toma en cuenta ciertas variables externas importantes como es la estacionalidad del sector y el nivel de competitividad que existe en el cantón Puerto Quito que es considerado amplio, por lo que, cualquier situación grave que la empresa no pueda sobrellevar, la competencia fácilmente podrá aprovecharse de esa situación, aspectos que están provocando que las ventas no tengan su ritmo normal. 


\section{BIBLIOGRAFÍA}

Banco Central del Ecuador. (2019). Obtenido de Sector maderero en Ecuador: https://blogs.cedia.org.ec/obest/wp-content/uploads/sites/7/2020/06/Sector-maderero-Ecuadoraprobado.pdf

Díaz, M., Parra, R., \& López, L. (2016). Enfoque para la planeación financiera. Colombia: Pearson Educación.

EL UNIVERSO. (2021). Recuperación de exportaciones y un acuerdo parcial, las expectativas del sector maderero para el 2021.

Fagilde, C. (2010). Presupuesto Empresarial. Bogotá: UNELLEZ.

Hair, J., Anderson, R., Mehta, R., \& Babin, B. (2010). Administración de Ventas. México: Learning.

Instituto Nacional de Contadores Públicos. (2012). Obtenido de INCP: https://incp.org.co/Site/2012/agenda/7-if.pdf

Instituto Nacional de Estadísticas y Censos. (2019). Obtenido de INEC: https://www.ecuadorencifras.gob.ec/documentos/web-

inec/Estadisticas_Economicas/DirectorioEmpresas/Directorio_Empresas_2019/Boletin_Tecnico_DIEE_ 2019.pdf

Jobber, D., \& Lancaster, G. (2012). Administración de Ventas. México: Pearson.

López, S. (2018). El presupuesto de ventas como herramiento de gestión. Escuela de Negocios y Dirección - Business Review, 40-55.

Luna Altamirano, K., Tinto Arandes, J., Sarmiento Espinoza, W., \& Cisneros Quintanilla, D. (2018). Implementación de un presupuesto empresarial base cero bajo el enfoque difuso. Universidad Estatal de Milagro, 4-9.

Moreno, T. (2019). El pronóstico de ventas en los negocios - modelos y aplicaciones. Santiago de Chile: Ril Editores.

Normas NIIF para las PYMES. (2020). Obtenido de Revisión Integral de la Norma NIIF para las PYMES: https://cdn.ifrs.org/content/dam/ifrs/project/2019-comprehensive-review-of-the-ifrs-for-smesstandard/rfi2020sp.pdf

Parra, J., \& Madriz, J. (2017). Presupuesto como instrumento de control financiero en pequeñas empresas de estructura familiar. Negotium, 35-46. doi:1856-1810

Pico, E., Suárez, K., \& Tomalá, C. (2017). La Planificación presupuestaria y su incidencia en la gestión financiera. Revista de Planeación y Control Microfinanciero, 1-10.

Plan de Desarrollo y Ordenamiento Territorial Pichincha. (2019-2022). Obtenido de GAD Pichincha: http://www.pichincha.gob.ec/phocadownload/LOTAIP_Anexos/Lit_K/pdot\%20actualizacion\%202015 $\% 20 \mathrm{al} \% 202019 . \mathrm{pdf}$ 
Rincón, C., \& Narváez, J. (2017). Presupuestos: Bajo Normas Internacionales de Información Financiera. Bogotá: Ediciones de la U.

Riquelme, M. (2017). Web y Empresas. Obtenido de https://www.webyempresas.com/presupuesto-deventas/

Rivadeneira, M. (2018). La Elaboración de Presupuestos en Empresas Manufactureras. En Presupuestos, Planificación y Control (págs. 41-42). México: Fundación Universitaria Andaluza Inca Garcillaso. Obtenido de https://www.eumed.net/libros-gratis/2014/1376/index.htm

Sánchez, A., Vaya, T., Mayorga, F., \& Freire, C. (2020). Sector maderero en Ecuador. Observatorio Económico y Social de Tungurahua.

Secretaría Nacional de Planificación y Desarrollo. (2018 - 2021). Obtenido de SENPLADES: https://www.planificacion.gob.ec/wp-content/uploads/downloads/2018/08/Plan-Estrategico-Senplades2018-2021.pdf

Trávez, S. (2020). Criterios Digitales. Obtenido de La Industria Forestal crece en medio de la crisis económica: https://criteriosdigital.com/portada/sloza/el-sector-maderero-crece-en-medios-de-una-crisiseconomica/

Valladares, C., Sánchez, K., Ugando, M., Sabando, A., \& Villalón, A. (2021). Gestión de capital de trabajo y su efecto en la rentabilidad para el grupo de empresas del sector manufacturero ecuatoriano. South Florida Journal Development. Miami, v. 2, n.2 apr./jun.p. DOI: https://doi.org/10.46932/sfjdv2n2-075 